\title{
SUCCINATE DEHYDROGENASE ACTIVITY IN UNEMBRYONATED EGGS, EMBRYONATED EGGS, MIRACIDIA AND METACERCARIAE OF SOME FLUKES
}

\author{
MINORU AKUSAWA \\ Department of Parasitology, Kurume University \\ School of Medicine, Kurume, Japan \\ KYOKO SAITO \\ Department of Chemistry, Nippon Veterinary and \\ Zootechnical College, Musashino, Tokyo, Japan
}

(Received for publication December 3, 1973)

\begin{abstract}
The authors examined the activity of succinate dehydrogenase (SD) in Fasciola hepatica and Paragonimus westermani with the stages in development from cell division egg to metacercaria. As a result of this experiment, in the unicellular stages and miracidium stages of F. hepatica and P. westermani, a very slight SD activity was detected. However. in the miracidium formation stages of these flukes, the activity was very strong. Thus, it was clear that the reduction of methylene blue decreased suddenly from the highest value when metamorphosis occurred. It was suggested that respiratory metabolism rises with the progress of development and ability to decolorize methylene blue in creases. In the metacercaria stages of $F$. hepatica, P.westermani, Metagonimus yokogawai, the ability to decolorize methylene blue was persistent, but it was not so strong. During the time, so called hibernation time, when metacercaria invades into final host, metacercaria keeps metabolism of respiration as low as possible, and saves consumption of energy for maintenance of life. Metacercaria has biological function for parasitism.
\end{abstract}

Biological studies have been made on the development of the flukes as specimens. Accordingly, all the life history has been clarified in these flukes up to this time. Especially, morphological studies have been performed in detail on the forms of the asexual stage of flukes. At present, it is clear that the flukes take a process of development starting with egg through miracidium, sprocyst, redia, and cercaria to metacercaria. And succinate dehydrogenase has been already found in some flukes (Huang and Chu, 1962 ; Bryant and Williams, 1962 ; Barry et al., 1968 ; Hamajima, 1972, 1973).

\section{MATERIALS AND METHODS}

\section{I ) Liver fluke eggs}

Bile samples were harvested from the gallbladder of a cow infected with Fasciola hepatica. Eggs were obtained from them by the sedimentation method. 


\section{II) Lung fluke eggs}

Metacercariae of Paragonimus westermani were isolated from Eriocheir japonicus, a kind of fresh-water crab, collected in Nagasaki Prefecture, and used to cause lung fluke infection in a dog. Adult worms were harvested from the lungs of the infected dog. Then fluke eggs were obtained from the uterus of these adult worms.

III) Cultivation and observation of egg:s

Collected eggs were washed well, treated with $5 \%$ antiformin, and washed again thoroughly with water.

The state of development of these eggs was observed every 5 days during the period of incubation. Materials were collected at the time of observation.

IV) Metacercaria

Succinate dehydrogenase activity in the stage of metacercaria was compared with some species of flukes. For this purpose, metacercariae of Metagonimus yokogawai were harvested from $G n a-$ thopogn elongatus elongatus, a kind of fresh-water fish, collected from the Tone River, and those of Heterophyes heterophyes were isolated from Triboloden hakonensis, another kind of freshwater fish, produced in Hida, Oita Prefecture.

V) Estimation of succinate dehydrogenase activity

The Thunberg tube was used to estimate succinate dehydrogenase (hereinafter referred to as SD) activity. The method of Tom and Wilson was applied to the measurement of ability to decolorize methylene blue.

The reaction mixture and Thunberg tube method is shown in Fig. 1. The substrate is sodium succinate. The test suspension is containing about $10^{4}$ eggs and $1 \%$ of worms prepared in each stage of development. Eggs were collected by centrifugation, placed in the

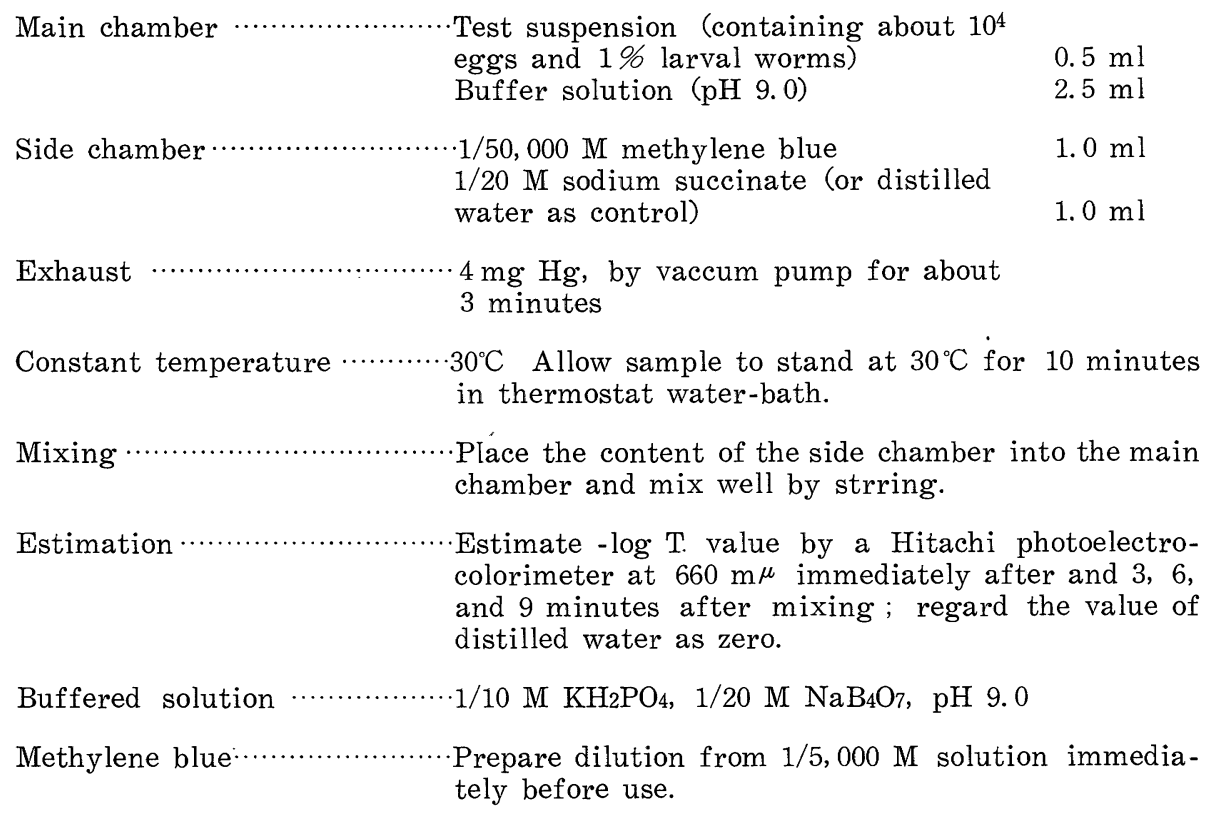

Fig. 1. Outline of the Thunberg tube method. 
glass homogenizer with addition of buffer solution, $\mathrm{pH}$ 9.0, and triturated in cooled condition. Such amount of buffer solution was added to the resulting sample as to make the total amount of the resulting suspension $5 \mathrm{ml}$. Then, $0.5 \mathrm{ml}$ of this suspension was put in the main chamber of the Thunberg tube as the test suspension. Again, $2.5 \mathrm{ml}$ of buffer solution was added to the content of the main chamber. In the side arm were placed $1.0 \mathrm{ml}$ of $1 / 50,000 \mathrm{M}$ methylene blue and then $1.0 \mathrm{ml}$ of $1 / 20 \mathrm{M}$ sodium succinate. After grease was painted, the tube was set with such care not to mix the content of the main chamber with that of the side arm. Then ventilation was induced by the vacum pump for about 3 minutes (at 4 $\mathrm{mg} \mathrm{Hg}$ ). The tube was allowed to stand in the thermostat at $30^{\circ} \mathrm{C}$ for $10 \mathrm{minu}$ tes, so that it might react and remain at a constant level of temperature.

Then the content of the side arm was mixed with that of the main chamber. The intensity of decoloration of methylene blue was estimated by the photoelectrocolorimeter immediately after 3, 6 , and 9 minutes after mixing of the contents.

\section{RESULTS AND DISCUSSION}

Experiment I. [Succinate dehydrogenase activity in unembryonated eggs, embryonated eggs, miracidia and metacercariae of liver fluke.]

It was not always possible to prepare homogeneous materials for measurement of the velocity of development of individual liver fluke eggs. Therefore, trials were repeated for this purpose. The results obtained are indicated in Table 1 and Fig. 2.

a) Unicellular stage

The extract obtained from $10^{4}$ eggs before cell division immediately after collection from the bile was examined
TABLE I

Ability to decolorize (liver fluke)

\begin{tabular}{l|c|c|c|c}
\hline & \multicolumn{4}{c}{- Log T } \\
\hline \multicolumn{1}{c|}{ Stage } & 0 & 3 & 6 & 9 \\
\hline Unicellular stage & 0.578 & 0.550 & 0.541 & 0.541 \\
Multicelllular stage & 0.553 & 0.531 & 0.525 & 0.496 \\
$\begin{array}{l}\text { Stage of miracidium } \\
\text { formation (A) }\end{array}$ & 0.593 & 0.553 & 0.516 & 0.490 \\
$\begin{array}{l}\text { Stage of miracidium } \\
\text { formation (B) }\end{array}$ & 0.660 & 0.603 & 0.590 & 0.580 \\
Miracidium stage & 0.563 & 0.547 & 0.547 & 0.544 \\
Metacercaria stage & 0.437 & 0.435 & 0.420 & 0.415 \\
\hline
\end{tabular}

for SD activity. As a result, the activity was first 0.578 , as expressed in terms of $-\log \mathrm{T}, 0.55$ after 3 minutes, 0.541 after 6 minutes, and 0.541 after 9 minutes. In short, changes in SD activity were very slight.

b) Multicellular stage

Egg's were incubated at $30 \mathrm{C}$ for 5 days. A number of multicellular eggs were observed under the microscope. The SD activity of these eggs showed a mild increase in ability to decolorize over a period from 6 to 9 minutes, as compared with the same activity of unicellular eggs.

c) Stage of miracidium formation (A)

Some eggs presented the miracidium formation during an incubation period of 10 days when it was observed by microscopy. As shown in Fig. 2-C, the ability to decolorize of the SD activity was 0.593 , as expressed in terms of -log $\mathrm{T}$, immediately after mixing the two contents, 0.553 , or 0.04 lower than the preceding value, after 3 minutes, and 0.553 after 6 minutes. In other words, the activity was not reduced, and decoloration taken place in the same inclination was still sharp after 9 minutes. The suspension retained only a very indistinct color of methylene blue after 2 hours. 

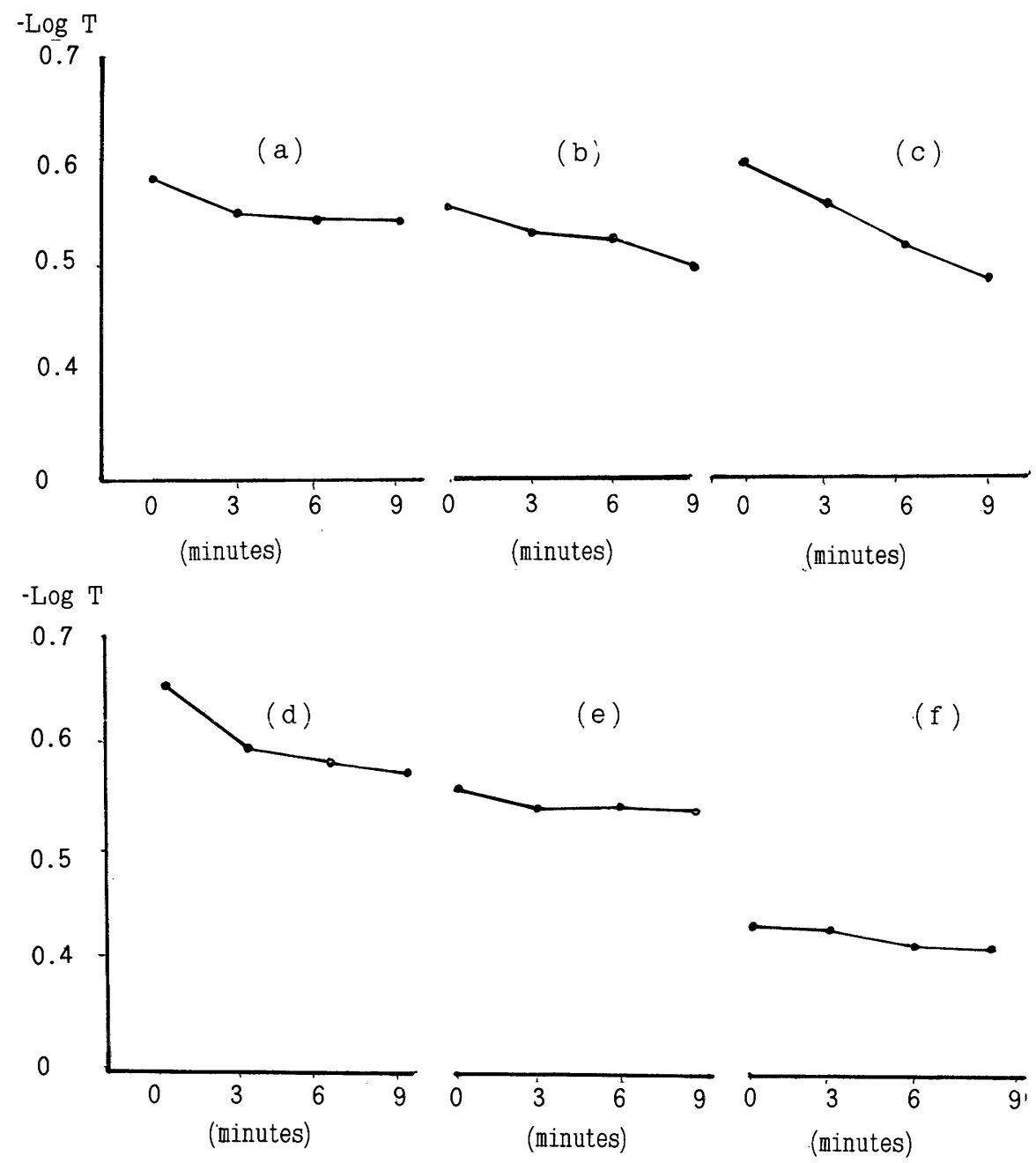

Fig. II Ability to decolorize (liver fluke)
(a) : Unicellular stage
(c) : Stage of miracidium formation (A)
(e): Miracidium stage

d) Stage of miracidium formation (B)

Some eggs were noticed to have formed miracidia. Eggs at 15 days of incubation are shown in Fig. II. Different from those at 10 days of incubation, they exhibited SD activity rapidly for the first 3 minutes, a little reduced activity after that, and a sudden dec- (b) : Multicellular stage

(d) : Stage of miracidium formation (B)

(f) : Metacercaria stage

rease in this activity over a period from 3 to 9 minutes.

e) Miracidium stage

A miracidium formed in the egg needs photo-energy to leave the egg. Therefore, the eggs were taken out of the incubator and exposed to light in order to collect miracidia. After some time, a number of miracidia could be recog- 
nized by the naked eye. When the cultures was confimed to contain miracidia released from eggs by microscopy, it was allowed to freeze rapidly in a freezing mixture composed of solid carbon dioxide and acetone, so that the miracidia might stop their movement. Then the frozen culture was thawed and centrifuged. The resulting material was used for the measurement. As shown in Figs. c and d, SD activity was very strong in eggs forming a miracidium. It was, however, extremely low in the miracidia formed, as shown in Fig. e.

\section{f) Metacercaria stage}

Cercariae released from Lymnaea snails were allowed to be settled on a sheet of cellophane, and the resultant metacercariae used as materials. Like miracidia, the metacercariae exhibited an ability hardly changeable to decolorize methylene blue. This ability, as expressed in terms of - log T, was recorded to be $0.437-0.435-0.42-0.415$ with the lapse of time. In Fig. II, comparison is made on the ability expressed in terms of - $\log \mathrm{T}$ to decolorize methylene blue measured sequentially at certin intervals over a period from the egg to the metacercaria of the liver fluke.

The results about the difference of ability to decolorize (SD activity) with

\section{TABLE II}

Difference of ability to decolorize (liver fluke)

\begin{tabular}{l|c|c|c|c}
\hline \multicolumn{1}{c|}{ Stage } & $0-3$ & $3-6$ & $6-9$ & Ave. \\
\hline Unicelluiar stage & 0.028 & 0.009 & 0.000 & 0.012 \\
Multicellular stage & 0.022 & 0.006 & 0.029 & 0.019 \\
$\begin{array}{l}\text { Stage of miracidium } \\
\text { formation (A) }\end{array}$ & 0.040 & 0.037 & 0.026 & 0.034 \\
$\begin{array}{l}\text { Stage of miracidium } \\
\text { formation (B) }\end{array}$ & 0.057 & 0.013 & 0.010 & 0.027 \\
Miracidium stage & 0.016 & 0.000 & 0.003 & 0.006 \\
Metacercaria stage & 0.002 & 0.015 & 0.005 & 0.007 \\
\hline
\end{tabular}

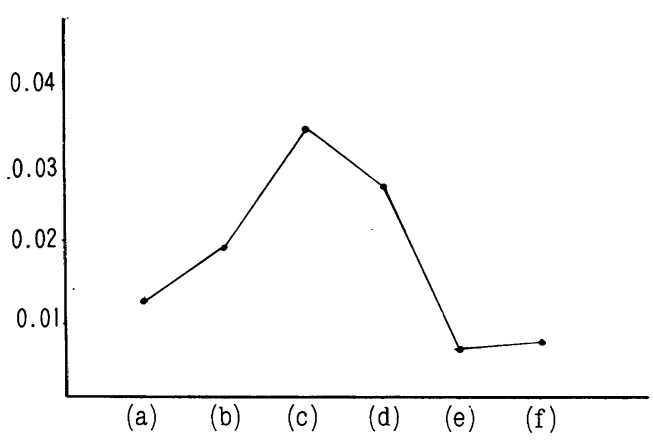

Fig. III Difference of ability to decolorize (liver fluke)

(a): Unicellular stage

(b) : Multicellular stage

(c) : Stage of miracidium formation (A)

(d) : Stage of miracidium formation (B)

(e) : Miracidium stage

(f): Metacercaria stage

the stage of development are indicated in Table II and Fig. III.

Experiment II. [Succinate dehydrogenase activity in unembryonated egg:s, embryonated eggs, miracidia and metacercariae of lung fluke.]

The lung fluke shows essentially the same stages of development as the liver

TABLE III

Ability to decolorize (lung fluke)

\begin{tabular}{l|c|c|c|c}
\hline \multicolumn{1}{c}{ Stage } & 0 & 3 & 6 & 9 \\
\hline Unicellular stage & 0.535 & 0.505 & 0.497 & 0.497 \\
Multicellular stage (A) & 0.735 & 0.645 & 0.610 & 0.540 \\
Multicellular stage (B) & 0.650 & 0.620 & 0.590 & 0.570 \\
Multicellular stage (C) & 0.715 & 0.670 & 0.620 & 0.590 \\
Multicellular stage (D) & 0.540 & 0.520 & 0.470 & 0.480 \\
Stage of miracidium & 0.485 & 0.475 & 0.425 & 0.375 \\
formation & 0.590 & 0.570 & 0.555 & 0.550 \\
Miracidium stage & 0.500 & 0.460 & 0.445 & 0.435 \\
Metacercaria stage & 0.50
\end{tabular}



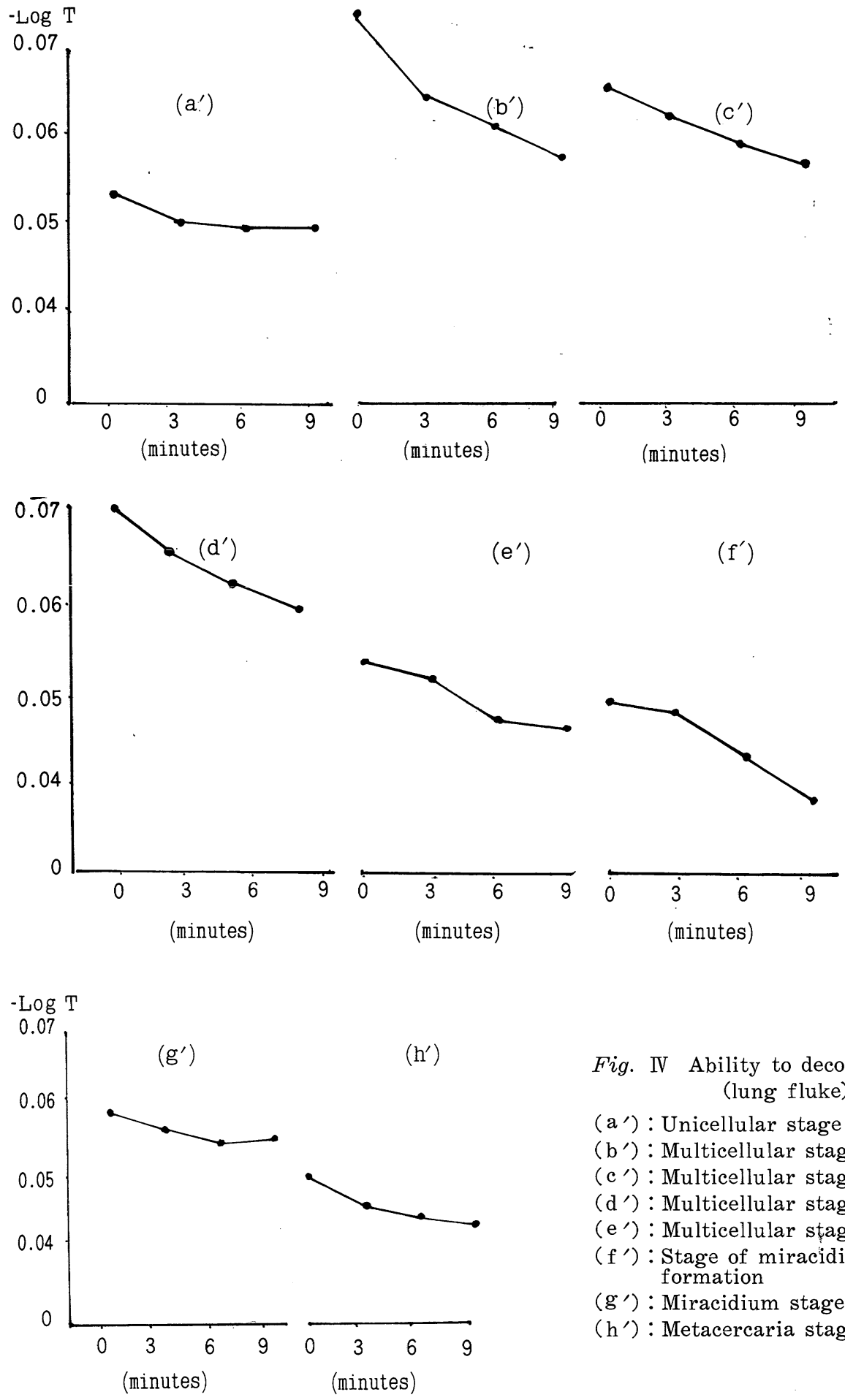

Fig. IV Ability to decolorize (lung fluke)

$\left(a^{\prime}\right)$ : Unicellular stage $\left(b^{\prime}\right)$ : Multicellular stage (A)

(c'): Multicellular stage (B)

$\left(d^{\prime}\right)$ : Multicellular stage (C)

$\left(\mathrm{e}^{\prime}\right)$ : Multicellular sțage (D)

$\left(f^{\prime}\right)$ : Stage of miracidium formation

$\left(\mathrm{g}^{\prime}\right)$ : Miracidium stage

$\left(h^{\prime}\right):$ Metacercaria stage 
fluke, but it is not parasitic on the same site as the latter. Essentially the same experiment as the preceding one was performed on lung flukes and gave the results presented in Table III and Fig. IV. As is clear from this figure, it was confirmed that lung flukes had shown SD activity similar to that of liver flukes.

a') Unicellular stage

Eggs were collected directly from the uterus of lung flukes and used as samples of the unicellular stage. The results obtained from them are shown in Table III and Fig. IV a'. They showed perfectly the same tendency as those obtained from liver flukes of the unicellular stage.

b') Multicellular stage

Egg's were incubated at $30^{\circ} \mathrm{C}$ for 5 days. The results obtained from them are presented in Table III and Fig. IV b'. SD activity was shown very remarkably over a period beginning immediately after the mixing of both contents at lasting for 3 minutes. During this period, the activity expressed in - log $\mathrm{T}$ value was very strong, ranging from 0.735 to 0.645 , with a difference of 0.09 .

c') Multicellular stage (A)

Eggs were incubated for 10 days. The results obtained from them are shown in Table III and Fig. IV c'. The ability to decolorize of these eggs was similar to that of liver fluke eggs incubated for 10 days.

d') Multicellular stage (B)

Miracidia were formed, without exception, in the liver fluke eggs incubated for 15 days, but not in any of the lung fluke eggs incubated for 15 days. As shown in Fig. IV d', SD activity was considerably stronger in the multicellular stage of eggs (B) than in that of eggs (A).

e') Multicellular stage (C)

Miracidia, which were not observed in stage d', failed to appear in this stage either when eggs were incubated for 20 days. As shown in Fig. IV e', the ability to decolorize, experessed to be $0.54 \rightarrow 0.52 \rightarrow 0.47 \rightarrow 0.46$. It decreased a little over a period from 6 to 9 minutes after the mixing of both contents. SD activity, however, was manifested distinctly.

f') Stage of miracidium formation

A large number of eggs forming miracidia were found in cultures on the 23rd day of incubation. As shown in Fig. IV f', SD activity was very strong. It was particularly strong over periods from 3 to 6 minutes and from 6 to 9 minutes. Therefore, SD activity was persistent.

\section{g') Miracidium stage}

Fig. IV g' indicates the result obtained from the miracidium stage. Materials for this stage were prepared by the same procedure as employed in the experiment on the liver fluke. As shown in Fig. IV g', eggs with formed miracidia exhibited the highest SD activity. Nevertheless, they presented such a remakable decrease in ability to decolorize as liver fluke.

h') Metacercaria

Fig. IV h' shows the results obtained from metacercariae of the lung fluke collected from a species of small freshwater crabs as materials. The metacercariae of the lung fluke exhibited a little stronger SD activity than those of the liver fluke, and stronger SD activity than the eggs of unicellular stage, but they presented a low value of ability to decolorize. This ability was persistent, although it remained low.

The difference of abillity to decolorize (SD activity) with the stages of development is indicated in Table IV and Fig. V. 
TABLE IV

Difference of ability to decolorize (lung fluke)

\begin{tabular}{l|c|c|c|c}
\hline \multicolumn{1}{c|}{ Stage } & $0-3$ & $3-6$ & $6-9$ & Ave. \\
\hline Unicellular stage & 0.030 & 0.008 & 0.000 & 0.013 \\
Multicellular stage (A) & 0.090 & 0.035 & 0.004 & 0.043 \\
Multicellular stage (B) & 0.030 & 0.030 & 0.020 & 0.027 \\
Multicellular stage (C) & 0.045 & 0.050 & 0.030 & 0.042 \\
Multicellular stage (D) & 0.020 & 0.050 & 0.010 & 0.027 \\
Stage of miracidium & 0.010 & 0.050 & 0.050 & 0.037 \\
formation & 0.020 & 0.015 & 0.005 & 0.013 \\
Miracidium stage & 0.040 & 0.015 & 0.010 & 0.022 \\
Metacercaria stage & & \multicolumn{3}{|c}{} \\
\hline
\end{tabular}

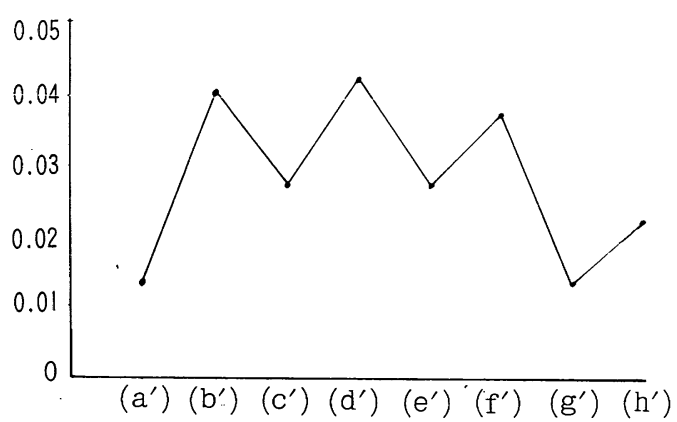

Fig. V Difference of ability to decolorize (lung fluke)

(a'): Unicellular stage

$\left(b^{\prime}\right)$ : Multicellular stage (A)

$\left(c^{\prime}\right)$ : Multicellular stage (B)

$\left(d^{\prime}\right)$ : Multicellular stage (C)

(e') : Multicellular stage (D)

$\left(f^{\prime}\right)$ : Stage of miracidium formation)

$\left(\mathrm{g}^{\prime}\right)$ : Miracidium stage

$\left(h^{\prime}\right):$ Metacercaria stage
TABLE V

Comparison of metacercaria stage ability to decolorize of flukes

\begin{tabular}{|c|c|c|c|c|}
\hline \multicolumn{5}{|c|}{$-\log \mathrm{T}$} \\
\hline Stage & 0 & 3 & 6 & 9 \\
\hline Fasciola hepatica & 0.437 & 0.435 & 0.420 & 0.415 \\
\hline $\begin{array}{l}\text { Paragonimus } \\
\text { westermani }\end{array}$ & 0.500 & 0.460 & 0.445 & 0.435 \\
\hline $\begin{array}{l}\text { Metagonimus } \\
\text { yokogawai }\end{array}$ & 0.575 & 0.575 & 0.560 & 0.540 \\
\hline
\end{tabular}
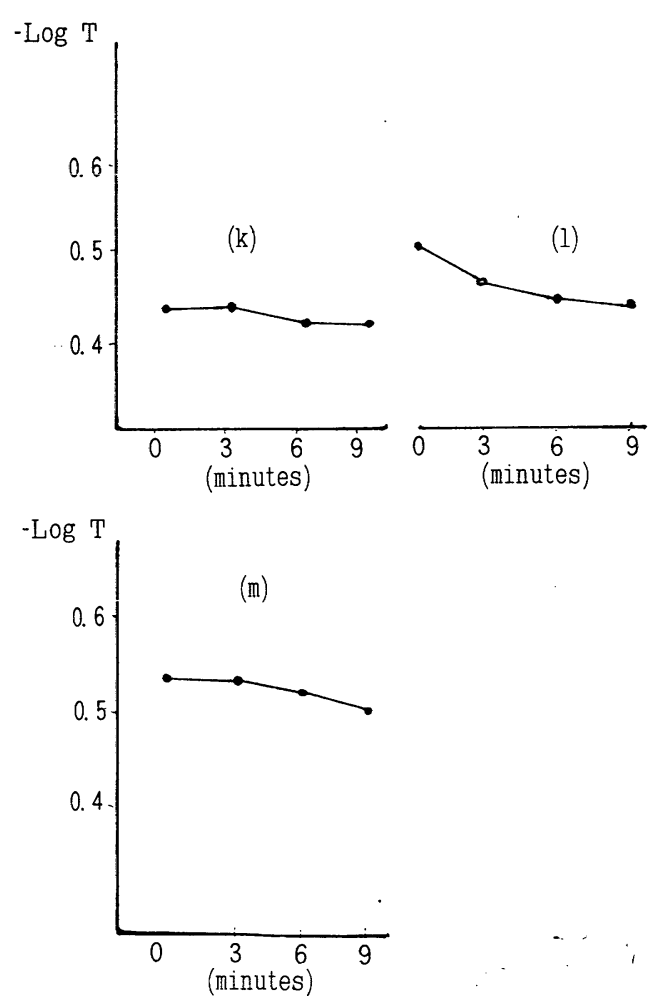

Fig. VI Comparison of metacercaria stage ability to decolorize of flukes.

(k): Fasciola hepatica

(1): Paragonimus westermani

(m) : Metagonimus yokogawai 
Experiment III. [Succinate dehydrogenase activity in metacercariae of Metagonimus yokogawai]

It was observed that there was a decrease in SD activity when metamorphosis occurred to the liver and lung flukes in the course of development. Metacercariae of Metagonimus yokoga$w a i$ were collected from scales of dece or Leuciscus hakonensis. The ability to decolorize methylene blue was measured in them and results obtained are indicated in Table V and Fig. VI. It was persistent, though low in value, in the metacercariae of both species.

Succinate dehydrogenase is important for oxidative metabolism. The activity is indicative of presence of tricarboxylic acid cycle and succinate oxidation. In this experiment, these results indicate that succinate dehydrogenase is present in unembryonated eggs, embryonated eggs, miracidia and metacercariae of these flukes as found in various life cycle stages of several trematode species (unembryonated eggs, embryonated eggs of $P$. westermani, Hamajima 1972, 1973; miracidia of $F$. hepatica, Bryant and Williams 1962; rediae and cercariae of Paragonimus ohirai, Hamajima 1973 ; metacercariae of Paragonimus miyazaki, Hamajima, 1972, 1973 ; adults of $F$. hepatica, Barry et al. 1968 and Bryant and Williams 1962 ; adults of Schistosoma mansoni, Huang and Chu 1962 ; adults of P.westermani, Hamajima 1973). In adition to the above mentioned, Bruce et al (1969) have shown a result of the experiment, suggesting presence of the dehydrogenase in cercariae of $S$. mansoni. Furthermore, in this experiment of $F$. hepatica and $P$. westermani, the rate of methylen blue reduction was higher in the miracidium formation stage eggs than in the unicellular stage eggs, miracidia and metacercariae. On the other hand, a lack of cytochrome oxidase activity was reported for Paragonimus unembryonated eggs (Hamajima 1973), and similar results were obtained from low $\mathrm{O}_{2}$ uptake and utilization of substrates in the homogenates of unembryonated eggs, miracidia, rediae and cercariae, and metacercariae of Paragonimus (Hamajima 1972). And also, metacercariae of Paragonimus resisted to cyanide inhibition (Hamajima 1973). Thus these results indicate that the physiological role of succinate oxidation is minor important in matacercaria stage. Moreover, it is suggested that the energy metabolism probably proceeds to the direction of the formation of pyruvate, phosphoenol pyruvate and succinate by malic enzyme, phosphoenol pyruvate carboxylase and fumarate reductase. On the contrary, the result obtained in this experiment for succinate dehydrogenase seems to indicate that the physiological role of succinate oxidation is more important to the miracidium formation stage eggs than unicellular stage eggs, miracidia and metacercariae becuase of the development and differentiation required large amount of energy. The activity in metacercearia of lung fluke was strong as compared with other flukes. This will be caused that metacercaria of lung fluke lives in Eriocheir japonicus.

\section{ACKNOWLEGDEMENT}

We wish to thank Prof. K. Okabe for many valuable suggestions made during the course of this work and also Dr. F. Hamajima for helpful advice and discussion.

\section{REFERENCES}

1) Barry, D. H., Mawclesley-Thomas, L. E., and Malone, J. C. : Enzyme histochemistry of the adult liver fluke, Fasciola hepatica. Experimental Parasitology 23, 355360, 1968.

2) Bruce, J. I., Weiss, E., Stirewalt, M. A., 
and Lincicome, D. R.: Schistosoma mansoni: Glycogen content and utilization of glucose, pyruvate, glutamate, and citric acid cycle intermediates by cercariae and schistosomules. Experimental Parasitology 26, 29-40, 1969.

3 ) Bryant, C., and Williams, J. P. G.: Some aspects of the metabolism of the liver fluke, Fasciola hepatica L. Experimental Parasitology 12, 372-376, 1962.

4) Hamajima, F. : Studies on metabolism of lung flukes genus Paragonimus V. Reaction of the tricarboxylic acid cycle in homogenates of eggs, larvae and adults. Japanese Journal of Parasitology 21, 280285, 1972.

$5)$ Hama jima, F.: Studies on metabolism of lung flukes of the genus Paragonimus VI. Succinoxidase system in homogenates of eggs, larvae, and adults. Experimental Parasitology 33, 515-521, 1973.

6 ) Huang, T. Y., and Chu, C. H. : Succinic oxidase of Schistosoma japonicum. Sheny Wu Hua Hsueh Yu Shing Wu Wu Li Hsueh Pao 2, 286-294; Chemical Abstracts 59, 14333-14334, 1962. 